\title{
ELECTRICAL TRANSPORT IN THICK FILM (CERMET) RESISTORS
}

\author{
MARIA PRUDENZIATI \\ Istituto di Fisica dell' Universitd di Modena, via Campi 213/A, 41100 Modena, Italy.
}

(Received May 10, 1982; in final form August 5, 1982)

Though for many decades have we had thick-film resistors (TFRs) with good electrical properties, no well established model for describing their conduction mechanisms has been achieved. However the efforts of some research groups in U.S.A. and Europe in the last years have had success in improving our knowledge of the microstructure and of a large spectrum of electrical properties of TFRs. The available information narrows the field of possibilities for conduction mechanisms in TFRs. Though no conclusive assessment is yet possible, some lines for further progress can be precisely delineated.

\section{INTRODUCTION}

It is not always necessary to understand the physics of a material in order to exploit its properties. This is the case for thick-film resistors (TFRs) whose applications largely exceed the development of a comprehensive model that describes their performances.

Attempts to formulate such a model date back to the beginning of thick-film technology with an incredible diversity of hypotheses on both the microstructure and transport mechanisms involved.

In some cases extreme importance was ascribed to the conductive phase, treated as a continuous sintered phase; also conductive grains sintered in chains and in ohmic contact were conjectured and thought to be responsible for the resistivity and its temperature coefficient (TCR). In other cases the dielectric (or glassy) phase was considered as a continuous uniformly-doped semiconductor responsible for most of the electrical properties of TFRs.

These models were proposed essentially because of the vague information available at that time on the microstructure of TFRs. Nowadays, this information is much more accurate and consequently the study of conduction mechanisms can be on a more sound basis, even if several problems are still being solved.

In this paper we will try to review briefly our present knowledge of the structural and electrical properties of TFRs and to discuss capabilities and limits of different models for conduction mechanisms in TFRs, consistent with these properties. Moreover some features which require further investigation will be enumerated and further work suggested.

\section{MICROSTRUCTURE OF THICK-FILM RESISTORS}

Extensive investigations ${ }^{1,2}$ have shown that the development of microstructure in TFRs is very complex and affected by a variety of parameters. The composition, softening point, viscosity, thermal expansion coefficient and wetting properties of the glass, the ratio of the size of glass particles to that of metal-oxide grains, ${ }^{2}$ and the sintering properties of the conductor material are some parameters known to affect the final microstructure of TFRs, besides the process condition (temperature and time in a defined firing cycle). Also 
we can expect differences in the structure, and of course in composition, between resistors obtained from inks of different formulation.

Nevertheless some general features emerge from the investigation of the structure of different resistors, which can be associated with the general features of their electrical properties.

The investigations can take advantage of optical inspections, X-ray diffraction, scanning electron microscopy (SEM), microprobe analysis and transmission electron microscopy (TEM). ${ }^{1-7}$ These techniques give complementary information for the characterization of TFRs, but owing to its resolution TEM gives the best results. ${ }^{4,5}$ The latter essentially show that the resistors are made up of a continuous glass phase with most of the conductive particles surrounded by glass (Figure 1). Conductive grains can differ largely in size, shape and reciprocal distance, and some clusters, generally built with the smallest grains, are present. In the case of ruthenate-pyrochlore-based resistors, ${ }^{5}$ the mean distance between grains is $50-100 \AA$ or more. At least locally, the dispersion of particles seems quite homogeneous. In fact the mean distance of grains observed is just the same that can be calculated for an homogeneous distribution by $s=d\left\{\left(\pi / 6 v_{c}\right)^{1 / 3}-1\right\}$ where the volume fraction of metal-oxide, $\mathrm{v}_{\mathrm{c}},{ }^{6}$ and the particle diameter, $\mathrm{d}$, are known.

However networks of the conductive phase have been frequently observed in optical micrographs ${ }^{1}$ and in TEM observations. ${ }^{4}$ Also ruthenate-pyrochlore-based resistors can be made of these networks as seen from TEM observations ${ }^{8}$ although this observation has not been included in the paper by Nordstrom and Hill. ${ }^{5}$

Energy dispersive X-ray spectra of very small areas of crystalline and amorphous phases give further interesting details on their compositions and show that, as a result of interface reaction of the metal-oxide grains with the matrix, Bi from bismuth-ruthenate partially exchanges with $\mathrm{Pb}$ from the glass and probably some $\mathrm{Ru}$ exchanges with $\mathrm{Si}^{5}$

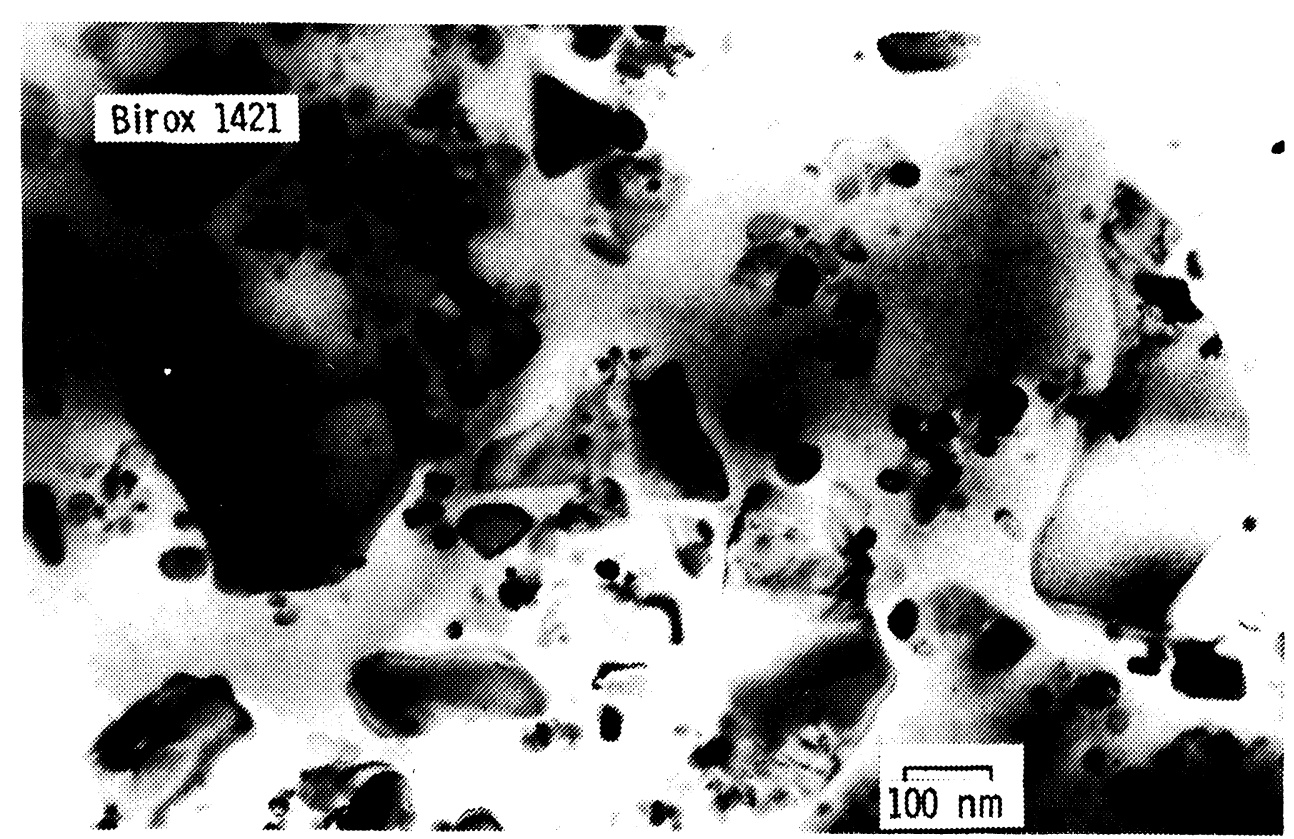

FIGURE 1 Transmission electron micrograph of fired sample of Birox 1421 material $\left(10^{2} \Omega / 0\right)$. After Nordstrom and Hills. ${ }^{5}$ 
Other results on grain and glass composition agree with previous information obtained with X-ray diffraction, microprobe analysis and X-ray fluorescence. ${ }^{3,7}$ Although it would be desirable to have TEM investigations on a larger variety of resistor systems and in resistors processed in different conditions, the reported results are a suitable basis to start a discussion on the identification of the conduction mechanisms in TFRs.

\section{CONDUCTION MECHANISMS AND ELECTRICAL PROPERTIES OF THICK- FILM RESISTORS}

The microstructure previously outlined indicates that the electrical path, even in lowresistivity TFRs, would require many places where conduction would be through the glass between grains (Figure 2).

The glass components used for TFRs are optically transparent and highly-resistive before the mixing with conductive particles. Then the glassy matrix should exhibit a high "energy gap" and a high potential barrier should exist between the oxide grains and the interparticle material, provided that no doping effect occurs during the mixing and firing processes. However interactions of the glass with metal-oxide are observed, so the question is to know the electrical effects of such interactions. We can suppose several possibilities; the interactions can lead to (Figure 3):

- shallow impurity levels which, in large concentration, bring the Fermi level $\mathrm{E}_{\mathrm{F}}$ of the system very close to the conduction band $\mathrm{E}_{\mathrm{c}}$ of the glass and make semiconduction in the glass a possible conduction mechanism (Figure 3a); this situation is seldom observed in glasses with a large energy gap;

- deep localized states in the band gap of the glass which add to the states, commonly observed in amorphous glasses due to imperfections, and to states arising from glassmodifiers and residues of organic vehicles. The mechanisms by which these localized states can affect the electrical properties of the glass are to provide states for electron hopping or to act as resonant centres for direct tunnelling of electrons from grain to grain (Figure 3b);

- a narrow band of delocalized electronic states far from the edges of the extended (conduction and valence) bands of the glass ${ }^{9}$ (Figure 3c).

No specific experiment has been performed in order to discriminate between these alternatives, but an analysis of the electrical properties of TFRs can help in the identification of the real situation. A brief review of these electrical properties is summarized in the following.

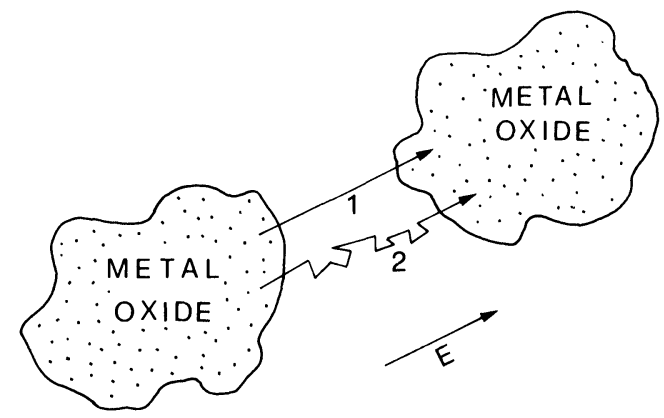

FIGURE 2 Schematic representation of the electron path between metal-oxide grains. The figure emphasizes the conduction through the glass with two alternatives. In case 1 electrons experience direct tunnelling between the grains, while in case 2 electrons "visit" some electronic states in the matrix. 


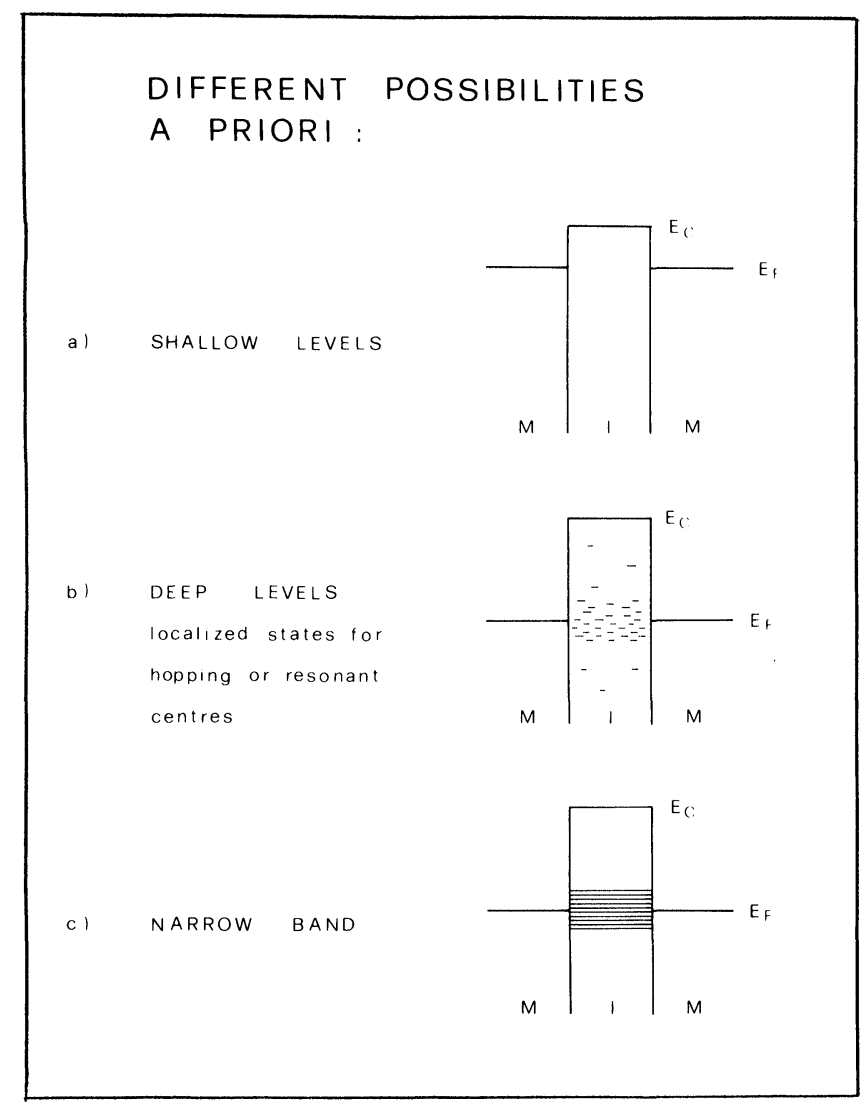

FIGURE 3 A sketch of the different a priori possibilities involved in the energetic situation in grains and intergranular material due to energy states created by imperfections and impurities in the insulating material. $M$ stands for metal oxide, I stands for dielectric material, $E_{F}$ represents the Fermi level of the system and $E_{c}$ the conduction band of the dielectric material. The electrical consequences of these different situations are discussed in the text.

The sheet resistivities of most widely used and studied TFRs are in a range from $10^{2}$ to $10^{6} \Omega / \square$ corresponding to conductivities of the films from $510^{-4}$ to $5 \Omega^{-1} \mathrm{~cm}^{-1}$ (for a film $20 \mu \mathrm{m}$ thick); this wide range of resistivities can be obtained by changing the volume fraction of the metal oxide from roughly $50 \%$ to $5 \% .^{6}$ Moreover the resistivity depends on the ratio between the size of the oxide grains to that of the original glass "spheres" in the paste (particle size effect). ${ }^{2}$ The resistivity of fired TFRs is very weakly temperature dependent, with TCR values of the order of some hundreds of $\mathrm{ppm} /{ }^{\circ} \mathrm{C}$ near room temperature and lower than $10^{4} \mathrm{ppm} /{ }^{\circ} \mathrm{C}$ at temperatures down to liquid nitrogen temperature; moreover the resistance exhibits a typical minimum often observed near room temperature; $;, 7,9,10$ also the TCR of TFRs exhibits a particle size effect. ${ }^{11}$ Moreover the resistance of TFRs is very weakly voltage dependent up to $10^{4} \mathrm{~V} / \mathrm{cm}{ }^{12}$ Thermoelectric effects give Seebeck coefficients of some tens of $\mu \mathrm{V} /{ }^{\circ} \mathrm{C} .^{6,13}$ Hall effect measurements allow us to obtain only an upper limit for the Hall mobilities (in the range from $10^{-3}$ to $10^{-2} \mathrm{~cm}^{2} / \mathrm{V} \mathrm{s}$ ) since the Hall signal is lower than the noise signal; ${ }^{6}$ the magnetoresistance is positive, very small and exhibits a linear dependence on the square of the magnetic field. ${ }^{6}$ The strain sensitivity of resistance in TFRs give gauge factors in the 
range from 2 to 15 according to different metal-oxides in the resistors. ${ }^{7}$ AC response at very high frequencies $\left(10^{6}-10^{11} \mathrm{~Hz}\right)$ shows a frequency dependence of both the real and imaginary parts of the impedence of TFRs. ${ }^{14}$ Moreover an a.c. component of the intergrain-material resistance $R_{b}$ is identified; this drops very rapidly with the frequency, a crude approximation being $R_{b}(\omega) \propto 1 / \omega$.

It is worth noticing that all these features have been observed in different series of commercial and custom resistors, i.e. in ruthenate-based as well as $\mathrm{RuO}_{2}-$ and $\mathrm{IrO}_{2}$-based resistors with glasses of different compositions.

If we take the alternatives previously enumerated into consideration in order to identify the conduction mechanisms in the intergranular material, we can conjecture that shallow impurity levels (low potential barriers) are unlikely in TFRs. In fact they should give rise to conduction processes generally characterized by very different temperature dependence of the resistance, particularly at very low temperatures, and by different Seeback coefficients, a.c. response and Hall effects than those observed in TFRs.

Only electronic levels with high activation energies in the gap of the glassy material have to be considered. The conduction mechanisms conceivable in this situation are:

- tunnelling of electrons between metal-oxide grains and localized states in glass and hopping transport in glass,

- direct tunnelling of electrons between nearest neighboring grains,

- conduction in a narrow band in the intergranular material.

We will examine now these possibilities and speculate about their implications for the electrical properties of thick-film materials.

\subsection{Hopping Transport}

There is no serious difficulty concerning the resistivity level of TFRs in a hopping model. Figure 4 shows the orders of magnitude of the conductivities of homogeneous glasses, in which conduction via hopping with a large concentration of localized states $\left(10^{19}-10^{20}\right.$ $\mathrm{cm}^{-3} \mathrm{eV}^{-1}$ ) near the Fermi level has been identified, ${ }^{15}$ in Figure 4 the range of conductivities of TFRs are also reported for comparison. It can be noted that there is a fairly good agreement between these values, except for the highest extreme of the conductivity range, but this is not surprising since we are comparing the electrical properties of "homogeneous" and very disordered materials.

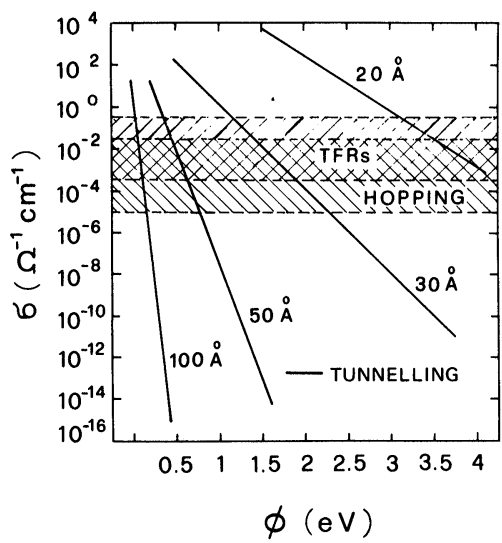

FIGURE 4 A comparison of orders of magnitude of conductivities for different materials (TFRs and glassy semiconductors) and tunnelling barriers with different potential barrier heights and thicknesses. 
We meet with more problems in the analysis of the temperature dependence of resistance, which in this case can be treated with a variable range hopping model ${ }^{\dagger 15}$ This gives:

$$
R=R_{0} \sqrt{ } T \exp \left(T_{0} / T\right)^{1 / 4}
$$

with

$$
\mathrm{T}_{0}=16 \alpha^{3} / \mathrm{NK}
$$

where $\alpha$ is defined such that $\exp (-\alpha \mathrm{x})$ is the rate at which the wave function of electrons falls off with the distance $\mathrm{x}$ from the localized centres; $\mathrm{N}$ is the number of localized states for unit energy and volume and $\mathrm{K}$ is the Boltzmann constant.

Linear plots of $1 \mathrm{n} R / \sqrt{ } \mathrm{T}$ vs $\mathrm{T}^{-1 / 4}$ have been obtained for resistors of different formulations and resistivities. ${ }^{16,17}$ The fitting is very good not only for temperatures lower than $T_{m}\left(T_{m}\right.$ is the temperature at which the resistance exhibits a minimum) but also at $\mathrm{T}>\mathrm{T}_{\mathrm{m}}$. This is surprising since the pre-exponential factor in Eq. (1) seems to derive from statistical considerations for $\mathrm{T} \rightarrow 0$, and should not give an increase of resistance at a finite temperature. Another reason for wondering if the fitting of Eq. (1) is fortuitous derives from the values of experimentally evaluated $T_{0}$ (in the range from $10^{3}$ to $10^{5} \mathrm{~K}$ ) which are too low with respect to the lower boundaries of the theory (around $10^{6} \mathrm{~K}$ ). ${ }^{18}$

However we feel that these arguments are not strong enough to definitely reject conduction processes in localized states in the gap of the intergranular material, although we suggest being cautious in applying a direct transposition of the usual formulae for hopping transport in the case of TFRs.

A simulative approach to the calculation of electrical transport in thick-film resistor has been presented, ${ }^{19}$ in which electrons are considered to hop from and to metallic grains and localized states in the glass. For concentrations of metallic grains sufficiently low and of localized states sufficiently high, a minimum in resistivity as a function of temperature is obtained. In fact at increasing temperatures, hopping is favoured, but when jumps are highly probable the influence of an external field which favours jumps in a given direction is less effective.

On the other hand an analysis of other transport properties of TFRs does not present real discrepancies with a hopping conduction mechanism in the intergranular material, characterized by a high density of localized states. Several similarities in the dependence of conductivity of TFRs on various experimental parameters are found with the same quantities in amorphous materials, in particular the a.c. response with the presence of a conductivity of the type $\sigma(\omega) \alpha \omega^{n}$ with $n \simeq 1$ is similar to that of amorphous materials. ${ }^{15}$

\subsection{Tunnelling}

In the framework of classical tunnelling models, which deal with a "perfect" dielectric free from substantial impurities, we can calculate ${ }^{20}$ that the conductivity level of TFRs is given either by very thin $(\simeq 20 \AA)$ high potential barriers or by thick and low barriers (see Figure 4). But previous considerations rejected the possibility of very low barriers

†This formulation was previously applied by Forlani and Prudenziati ${ }^{10}$ to percolative tunnelling of electrons between metal-oxide grains with no contribution of localized states in the intergranular material; this approach is not correct in this case since only nearest neighbouring grains are accessible for electrons in this picture. 
while TEM experiments have shown that the geometrical separation of the grains is generally from 50 to $100 \AA$ or more. Consequently tunnelling processes can give substantial contribution to the conductivity of TFRs only if it is greatly enhanced by resonant centres in the intergranular material. Indeed experimental investigations on simple MIM structures $^{21}$ have shown that impurities in the insulator can increase the conductivity of the barrier by orders of magnitude or in other words can make the "effective barrier thickness", s', lower than the geometrical thickness, s.

Within this framework Pike and Seager ${ }^{6}$ proposed that the minimum of the resistance in TFRs can be ascribed to a tradeoff between a negative temperature coefficient of resistivity, given by the tunnel process combined with the electrostatic energy required to transfer an electron from a neutral grain to another grain, and a positive temperature coefficient, provided by metal-oxide grains. According to their analysis, the presence of a minimum of resistance in ruthenate-pyrochlore-based resistors at the observed temperature would require a contribution of the resistivity due to the grains of the order of $30 \%$ independently of the sheet resistivity of a series of resistors, ranging from $10^{2} \Omega / \square$ up to $10^{6} \Omega / \square$. This proposal seems to be supported by the fact that TFRs, made with even weakly semiconducting particles, never show a resistivity minimum, ${ }^{8}$ but some questions remain open. Firstly it is puzzling why the resistance of grains should scale in the same way as that of the resistance of the tunnelling barriers, so that the contribution of the grains to the total resistivity remains fixed. Moreover we should expect that the temperature coefficients of resistance (TCR) in $\mathrm{IrO}_{2}$ and $\mathrm{RuO}_{2}$-based resistors would be very different from that of pyrochlore-based resistors, since the TCR of Ir and Ru dioxides are much greater ${ }^{20}$ than that of ruthenate pyrochlores. ${ }^{23}$

Finally, measurements of the high frequency response of TFRs do not support the hypothesis of such a great contribution to resistance due to grains. ${ }^{14}$

An alternative proposal for the origin of the minimum of the resistance in TFRs in the framework of resonance-assisted tunnelling transport is that the tunnelling barrier height $\Phi$ is temperature dependent, increasing when the temperature increases. It is found that very low temperature coefficients of $\Phi$ (around $20-50 \mathrm{ppm} /{ }^{\circ} \mathrm{C}$ ) are enough to provide the minimum of resistance and give a very good fitting of experimental data over large temperature range (from 77 to $600^{\circ} \mathrm{K}$ ). ${ }^{24}$

\subsection{Conduction in a Narrow Band}

In order to describe the temperature dependence of resistance in TFRs, an alternative model has been proposed ${ }^{9}$ which requires a very narrow $\left(10^{-4} \mathrm{eV}\right)$ band of states in the inter-granular material; they are supposed to be due to transition metal atoms diffused from the grains into the glass matrix, during the firing process of the resistors. The atoms are assumed to bond with the oxygen atoms of the silica network and so form $\pi^{+}$ bonds of the same type which give rise to narrow conduction bands in transition metal oxides. ${ }^{25}$ At sufficiently high concentration $\pi^{+}$bonds overlap to form a narrow band of states which could be half-filled. At low temperatures electrons of poorly connected impurity centres will be trapped and will not contribute to the formation of an electron gas so that the material will look insulating. At high temperatures they can contribute to drive the matrix into a metallic form, though very "weakly metallic". This model has not been further developed in any analytical formulation so, even if suggestive, it eludes any quantitative check. However it could be consistent with very low Hall mobilities and Seebeck coefficients as well as low magnetoresistance effects.

From the point of view of the chemistry required, interface reactions of $\mathrm{Ru}$ and $\mathrm{Bi}$ from ruthenate-grains with the glassy matrix are largely documented by energy dispersive $\mathrm{X}$-ray spectra in TEM experiments. However we do not know if the concentration involved is large enough to support the formation of the suggested narrow bands. 
It could be interesting to examine more deeply the implications of this model both from the theoretical point of view (electrical consequences of a narrow band) and the experimental point of view (e.g. analysis of interface reactions in $\mathrm{RuO}_{2}$ and $\mathrm{IrO}_{2}$-based resistors).

\section{CONCLUSIONS}

Recent experimental investigations on the microstructure, composition and electrical properties of TFRs have provided valuable information and allowed of progress in the knowledge of these materials. However the identification of the predominant conduction mechanism is not yet definitive. We can now focus our attention on a few alternatives and reject other ones that have been discussed. All the interesting possibilities for conduction mechanisms in TFRs suggest that simple and classical transport models are inappropriate for a complete description of the electrical properties of TFRs. The alternatives are conduction mechanism by hopping, tunnelling or conduction in narrow bands, and in all cases a relevant role is to be played by deep energy levels introduced in the intergranular material of TFRs during the processes required to prepare the resistors.

These conclusions could sound quite obvious to people who, being engaged in the formulation of inks, for a long time have observed how sensitive the electrical properties of TFRs are to glass modifiers and the specific properties of the constituent used. On the contrary people involved in the interpretation of the electrical properties of TFRs seem less aware of the relevance of composition of the intergranular material.

For a long time attempts to apply classical transport models to TFRs have been made; the most recent results and speculations suggest new approaches to the problem with more emphasis on studies specifically tailored to TFRs.

Several areas of investigation can be conceived for further progress in the development of a conduction model for TFRs. More extensive experiments on the microstructure of TFRs and on the composition of the intergranular material could be valuable; investigations on the electrical properties of structures more simple than TFRs but with the same "compositional ingredients" could be informative. A more systematic study on electrical noise and its temperature dependence ${ }^{26,27}$ could provide further information.

More investigations on the possible existence of a narrow conduction band created by reactions of transition metal atoms with the glassy matrix of TFRs and on the electrical consequences of this band would be interesting, as well as a more precise approach to tunnelling processes assisted by resonant centres.

However some of these investigations would require a certain level of sophistication in the theoretical and experimental methods and then a definitive conclusion on a comprehensive conduction model of TFRs could require a long time.

\section{ACKNOWLEDGEMENTS}

The author wishes to thank Profs. C. Jacoboni, Drs. L. Reggiani and B. Morten for stimulating discussions. This work was partially supported by Consiglio Nazionale delle Ricerche of Italy.

\section{REFERENCES}

1 R.W. Vest, "Conduction mechanisms in thick-film microcircuits" Purdue Final Technical Report, Arpa Order 1642, 1975 unpublished. 
2 Ching Tzuen Tarn, "Particle size effects on electrical properties of thick-film resistors" M.S. Thesis, Purdue University 1979 , unpublished.

3 J.S. Shah and W.C. Hahn, Electron. Comp. Conf. 234, Anheim, U.S.A. (1978).

4 D.J. Pedder, Proc, 1st Europ. I.S.H.M. Conf. Bad Homburg, paper XXX (1977).

5 T.V. Nordstrom and C.R. Hills, Proc, 1979 Int. Microelectronic Symposium, Los Angeles p. 40 (1979).

6 G.E. Pike and C.H. Seager, J. Appl, Phys, 48, 5152 (1977).

7 C. Canali, D. Malavasi, B. Morten, M. Prudenziati and A. Taroni, J. Appl. Phys. 51, 3282 (1980).

8 G.E. Pike, Private communications.

9 R.M. Hill, Proc. 2nd Europ. I.S,H.M. Conf., Ghent p. 95, (1979).

10 F. Forlani and M. Prudenziati, Electro. Comp. Sci. Technol, 3, 77 (1976).

11 A.H. Boonstra and C.H. Mutsaers, Thin Sol. Films 67, 13 (1980).

12 J. Robertson, Proc. 1st Europ. I.S.H.M. Conf., Bad Homburg, paper XXI (1977).

13 M. Prudenziati and A. Cattaneo, Electro Comp. Sci. Technol. 3, 181 (1976).

14 M. Prudenziati, B. Morten, C. Martini and G. Bisio, in Recent Development in Condensed Matter Physics, Vol. 2, J.T. Devreese, L.F. Lemmens, V.E. Van Doren and J. Van Royen Eds, (Plenum Press, New York, 1981) p. 399.

15 M.F. Mott and E.A. Davis, Electronic Processes in Non-Crystalline Solids (Oxford University Press, Oxford 1975).

16 M. Prudenziati, Alta Frequenza 46, 287 (1977).

17 A. Cattaneo, M. Cocito, F. Forlani and M. Prudenziati, Electro Comp. Sci. Technol. 3, 205 (1977).

18 V. Ambegaokar, B.I. Halperin and J.S. Langer, Phys. Rev. B-4, 2612 (1971).

19 C. Jacoboni, M. Prudenziati and A. Rizzi, Proc, 3th Europ. I.S.H.M. Conf., Avignon p. 11, (1981).

20 J.G. Simmons, J. Appl. Phys. 34, 1973 (1963); J. Appl, Phys. 35, 2655 (1964).

21 J.C. Fisher and I. Giaever, J. Appl. Phys. 32, 172 (1961).

22 W.D. Ryden, A.W. Lawson, C.C. Sartain, Phys, Lett. 26A, 209 (1968).

23 P.R. Van Loan, Ceram. Bull. 51, 231 (1972).

24 M. Prudenziati, A. Rizzi, P. Davoli and A. Mottei to be published.

25 J.B. Googenough, J. Appl. Phys. 37, 1415 (1966).

26 T.M. Chen and D. Smith, Proc. 2nd Int. Symposium on 1/f Noise, Florida 1980, 376.

27 M. Prudenziati, B. Morten and A. Masoero, 6th Int. Conf. on Noise in Physical Systems, U.S. Dept. of Commerce, National Bureau of Standards, NBS Publication 614, p. 202-205. 

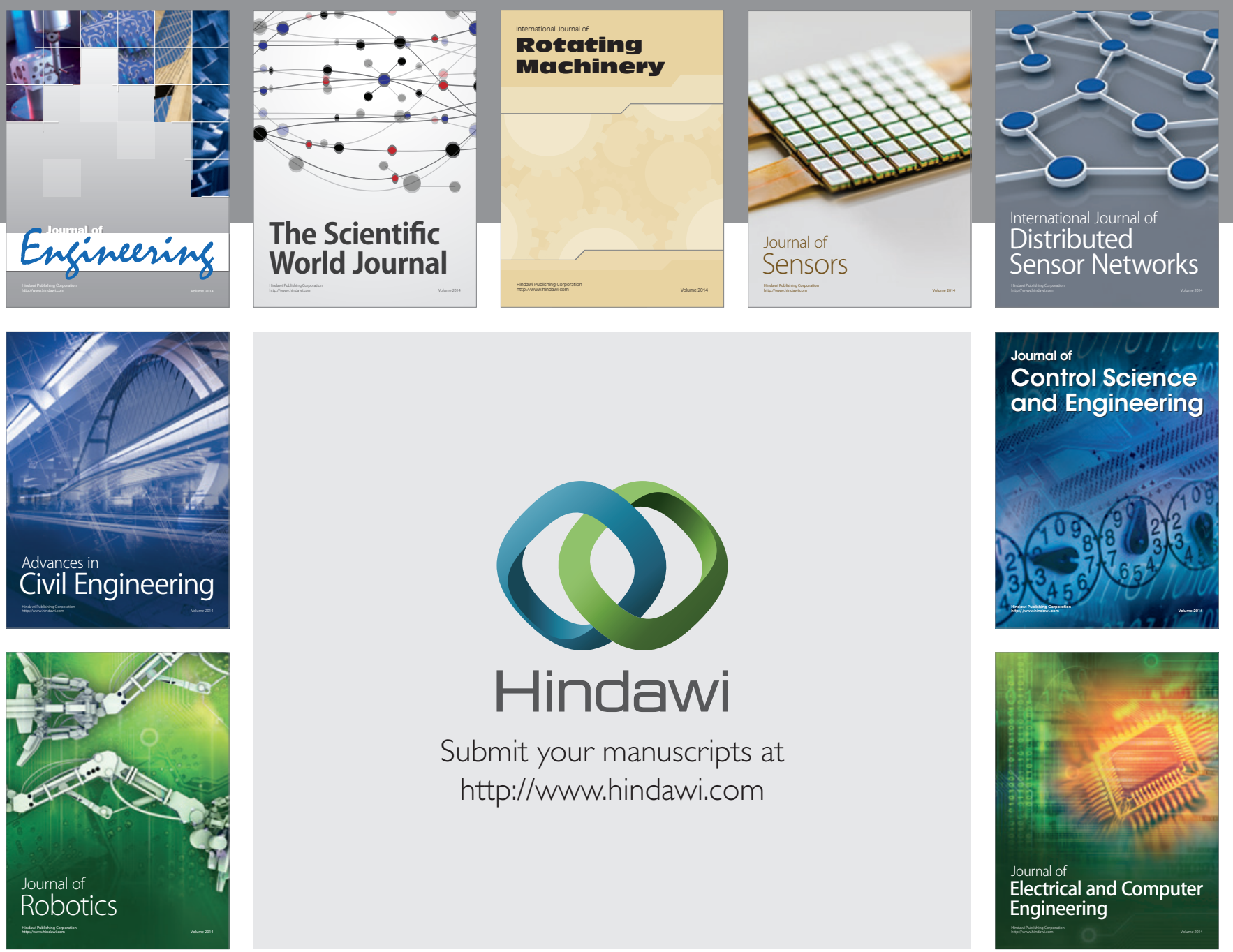

Submit your manuscripts at

http://www.hindawi.com
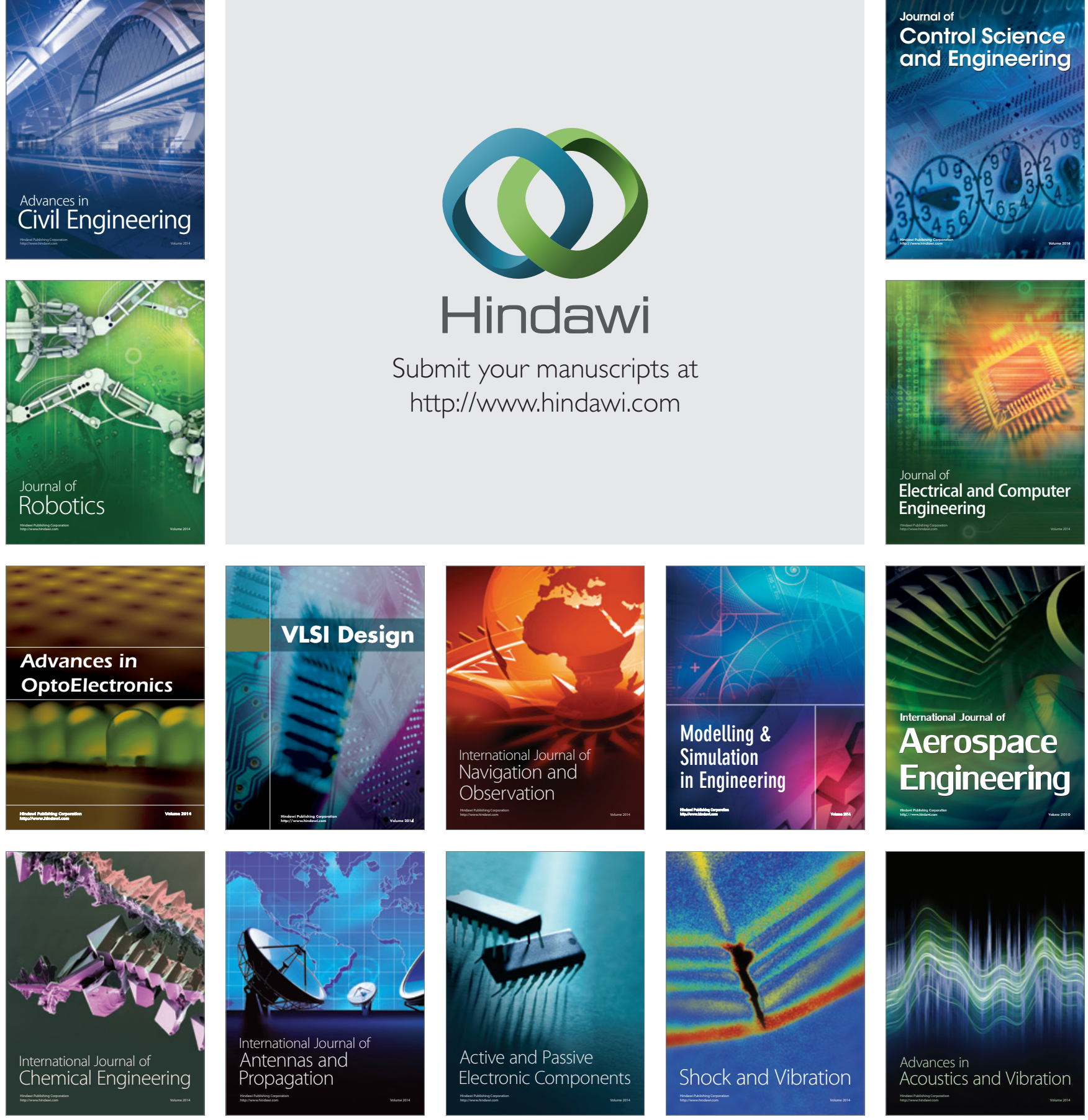